\title{
Role of the imprinted allele of the Cdkn1c gene in mouse neocortical development
}

\begin{abstract}
Yui Imaizumi ${ }^{1}$, Shohei Furutachi ${ }^{1,2}$, Tomoyuki Watanabe ${ }^{1}$, Hiroaki Miya ${ }^{1}$, Daichi Kawaguchi ${ }^{1 *}$ \&Yukiko Gotoh $\mathbb{1}^{1,3^{*}}$

Imprinted genes are expressed from only one allele in a parent of origin-specific manner. The cyclindependent kinase inhibitor $\mathrm{p} 57^{\mathrm{kip2}}$ is encoded by an imprinted gene $C d k n 1 c$, with the paternal allele being silenced. The possible expression and function of the paternal allele of $C d k n 1 c$ have remained little studied, however. We now show that the paternal allele of the $C d k n 1 c$ gene is expressed at a low level in the developing mouse neocortex. Surprisingly, the central nervous system-specific conditional deletion of the paternal allele (pat cKO) at the Cdkn1c locus resulted in a marked reduction in brain size. Furthermore, pat cKO gradually reduced the number of neural stem-progenitor cells (NPCs) during neocortical development, and thus reduced the number of upper-layer neurons, which were derived from late-stage NPCs. Our results thus show that the paternal allele of the $C d k n 1 c$ locus plays a key role in maintenance of NPCs during neocortical development.
\end{abstract}

Genomic imprinting refers to an epigenetic process that results in the inactivation of one of the two alleles of a gene in a parent of origin-dependent manner ${ }^{1-3}$. It is achieved mainly by allele-specific DNA methylation at a subset of CpG islands, known as imprinting control regions (ICRs), during early developmental stages ${ }^{4}$. Imprinted genes play essential roles in development, homeostasis, and behavior in mammals ${ }^{5,6}$. Changes at imprinted gene loci in humans are associated with diseases such as Beckwith-Wiedemann syndrome, Prader-Willi syndrome, and Angelman syndrome ${ }^{7,8}$, many of which are characterized by altered growth and mental disorders ${ }^{6}$. Although canonical genomic imprinting has been thought to result in the complete silencing of one allele of a gene, recent studies have shown that silencing of some imprinted genes appears to be incomplete or reversed to various extents in the brain ${ }^{9}$. For instance, derepressed expression of the imprinted alleles of $I g f 2$ and $D l k 1$ contributes to the regulation of adult neural stem cells in mice $^{10,11}$. However, the functions of the imprinted alleles of other genes remain mostly obscure.

The Cdkn1c gene is imprinted, with the maternal allele being expressed, and is located in the distal region of mouse chromosome 7 and human chromosome $11 \mathrm{p} 15^{12,13}$. In mice, DNA methylation of two ICRs, KvDMR ${ }^{14-16}$ and ICG $5^{17}$, has been suggested to suppress expression of the paternal allele of the $C d k n 1 c$. The Cdkn1c-encoded protein $\mathrm{p}^{\mathrm{kip} 2}$ is a cyclin-dependent kinase inhibitor $(\mathrm{CKI})^{18,19}$ that is highly expressed in neural and skeletomuscular tissues $^{20,21}$ during embryonic development. In humans, changes at the $C d k n 1 c$ gene locus are associated with Beckwith-Wiedemann syndrome, features of which include excessive growth and an increased risk of childhood cancer $^{22,23}$. Gene knockout $(\mathrm{KO})$ studies have implicated $\mathrm{p} 57^{\mathrm{kip} 2}$ in regulation of fetal growth and placental development $^{20,24,25}$. In the central nervous system (CNS), $\mathrm{p} 57^{\text {kip2 }}$ plays a key role in regulation of the proliferation and differentiation of embryonic neural stem-progenitor cells (NPCs) and adult neural stem cells ${ }^{26-33}$. CNS-specific conditional KO of the maternal Cdkn1 $c$ allele resulted in the induction of cell death in a manner dependent on the transcriptional regulators E2F1 and p53, thinning of the neocortex, and pronounced hydrocephalus in mice, with the latter effects possibly reflecting a function of $\mathrm{p}^{2 \mathrm{kip} 2}$ in the subcommissural organ (SCO $)^{30}$.

The paternal allele of the $C d k n 1 c$ gene has been thought to be completely silenced, given that conventional $\mathrm{KO}$ of the maternal allele appeared to result in elimination of $C d k n 1 c \mathrm{mRNA}$ and protein ${ }^{20}$ and that a reporter for the paternal allele did not show any expression during normal development unless challenged by stress ${ }^{34}$. Indeed, the phenotype of conventional maternal $C d k n 1 c$ KO mice appears essentially identical to that of conventional

${ }^{1}$ Graduate School of Pharmaceutical Sciences, The University of Tokyo, Tokyo, 113-0033, Japan. ${ }^{2}$ Sainsbury Wellcome Centre for Neural Circuits and Behaviour, University College London, London, W1T 4JG, UK. ${ }^{3}$ International Research Center for Neurointelligence (WPI-IRCN), The University of Tokyo, Tokyo, 113-0033, Japan. *email: dkawaguchi@ mol.f.u-tokyo.ac.jp; ygotoh@mol.f.u-tokyo.ac.jp 
a

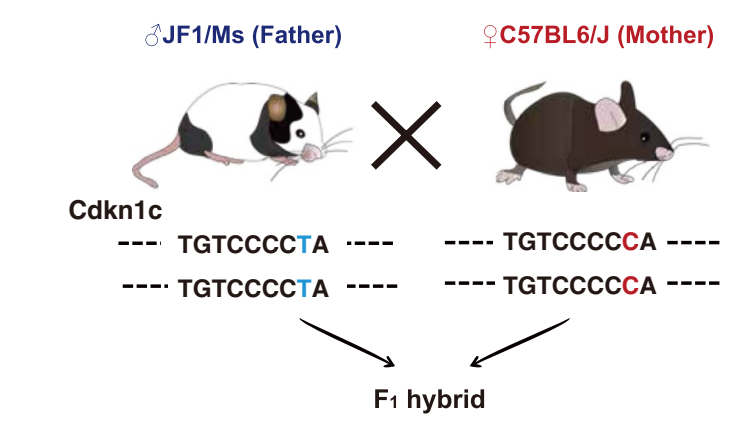

Paternal Cdkn1c (JF1/Ms)

TGTCCCCTA

Maternal Cdkn1c (C57BL/6J) b

Paternal (JF1) /Maternal (BL6) Cdkn1c expression ratio

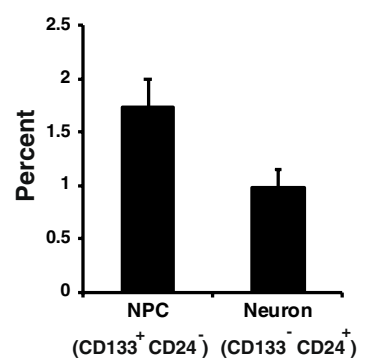

d

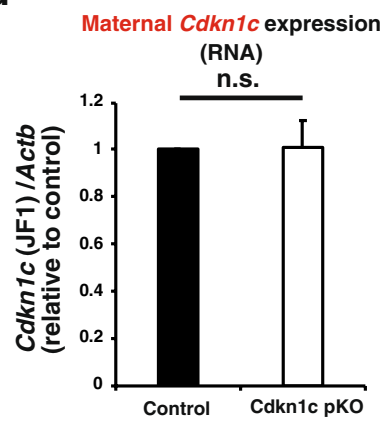

C

Paternal Cdkn1cexpression (RNA)

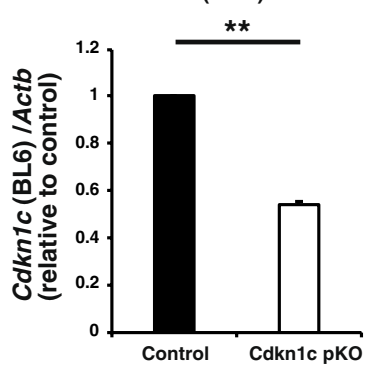

e

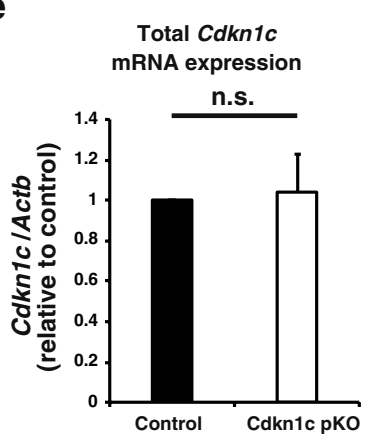

Specific primers for maternal or paternal allele

Figure 1. Detection of Cdkn1c mRNA expression from the paternal allele in the embryonic neocortex. (a) Scheme of allele specific qPCR of Cdkn1c. F1 hybrid embryos of C57BL/6J (BL6) female and JF1/MS (JF1) male parents have SNPs derived from their parents. qPCR was performed by the use of specific primers for each allele. (b) The ratio of paternal to maternal Cdkn1c mRNA expression in NPCs and in neurons was determined by allele specific qPCR under the hybrid (JF1 male and BL6 female) genetic background at E16. FACS was used to isolate NPCs (CD133 ${ }^{+} \mathrm{CD} 24^{-}$population) and neurons (CD133- ${ }^{-} \mathrm{CD} 24^{+}$population). (c,d) Allele specific qPCR of $C d k n 1 c$ mRNA in the neocortex isolated from control (Cdkn1c pat flox (BL6)/+(JF1) $)$ and hybrid paternal $C d k n 1 c$ cKO (Nestin-Cre; $C d k n 1 c^{\text {pat flox(BL6)/+(JF1) }}$ mice at E16. Cdkn1c mRNA expression from each allele was normalized to $\beta$-actin. Data are expressed relative to the corresponding value for control mice. (e) qPCR of total $C d k n 1 c$ mRNA expression in the neocortex isolated from control and paternal $C d k n 1 c \mathrm{cKO}$ mice in the $\mathrm{BL} / 6 \mathrm{~J}$ background at P0. Cdkn1c mRNA expression was normalized to $\beta$-actin. Data are expressed relative to the corresponding value for control mice. Data are mean + s.e.m from three independent experiments. Paired twotailed Student's t-test; $* * P<0.01$. n.s., not significant.

null Cdkn1c KO mice $e^{20,24,32}$, and paternal Cdkn1c KO mice have been found to manifest no distinct phenotype $\mathrm{e}^{24}$. These observations have thus suggested that only the maternal allele of the Cdkn1c gene locus is indispensable. However, it has remained possible that the paternal allele of this locus is transcribed at a low level, and the necessity of the paternal allele for development has remained poorly explored.

Here we examined the expression and functional importance of the paternal Cdkn1c allele in the developing mouse brain. Reverse transcription (RT) and allele-specific quantitative polymerase chain reaction (qPCR) analysis revealed that the paternal allele is indeed expressed at a low level in embryonic NPCs. Unexpectedly, CNS-specific paternal Cdkn1c KO resulted in a substantial reduction in brain size, with the number of upper layer neurons in the neocortex showing a marked decrease. Consistent with this latter observation, the number of NPCs that give rise to upper layer neurons was also decreased in the mutant mice. Our findings thus uncover an essential role for the imprinted allele of the Cdkn1c gene in the developing nervous system.

\section{Results}

Expression of the paternal Cdkn1c allele in neocortical NPCs and neurons during mouse embryonic development. We first examined whether expression of the paternal (imprinted) allele of the Cdkn1c gene could be detected in the developing brain with the use of chimeric mice derived from a cross between lines-C57BL/6J (BL6) and JF1/Ms (JF1) - that differ with regard to single nucleotide polymorphisms (SNPs) at this locus. We thus collected $\mathrm{F}_{1}$ hybrid embryos at embryonic day (E) 16 from a cross between JF1 male and BL6 female mice. Total RNA isolated from the embryonic neocortex was then subjected to RT and allele-specific qPCR analysis with primers designed to recognize individual SNPs (Fig. 1a). In contrast to previous results showing that the paternal allele of the $C d k n 1 c$ gene is completely silenced ${ }^{20,34}$, we detected expression of the paternal allele at a level corresponding to $\sim 1 \%$ to $2 \%$ of that of the maternal allele (Fig. 1b). We did not detect 
Cdkn1c mRNA in embryos from a cross between BL6 male and BL6 female mice with the use of JF1-specific primers or in those from a cross between JF1 male and JF1 female mice with the use of BL6-specific primers, confirming the specificity of the line-specific primers. We also performed the reciprocal cross (BL6 male with JF1 female) and examined offspring for expression of the maternal and paternal Cdkn1c alleles by RT-qPCR analysis. The expression level of the paternal allele relative to that of the maternal allele was almost the same as that for offspring of JF1 male with BL6 female (Supplementary Fig. 1a), indicating that this paternal expression was not due to a strain specific effect. Furthermore, the paternal and maternal $C d k n 1 c$ expression levels reported in the previous RNA sequencing analysis in the cortex at E13 and P0 are consistent with our results ${ }^{35}$. We next deleted the paternal allele of the Cdkn1c gene in the CNS by crossing JF1 female mice heterozygous for a Cre recombinase transgene under the control of the Nestin enhancer (Nestin-Cre) with BL6 male mice harboring floxed alleles of the $C d k n 1 c$ gene $\left(C d k n 1^{\mathrm{f} / \mathrm{fl}}\right)$ (Fig. $1 \mathrm{c}$ and Supplementary Fig. 1d). Expression of the paternal Cdkn1c allele in the neocortex was partially but significantly reduced in the resulting conditional $\mathrm{KO}$ (cKO) embryos at E16 compared with control littermates lacking the Nestin-Cre transgene (Fig. 1c). Deletion of the maternal Cdkn1c allele by the Nestin-Cre reduced the expression of maternal Cdkn1c to a similar level with that observed in the paternal cKO (Supplementary Fig. 1b,c). The partial reduction of paternal and maternal Cdkn1c transcripts by these cKO is likely due to those expressed before the expression of Nestin-Cre, which starts to be expressed in the CNS from $\sim \mathrm{E} 11^{36}$. This result thus suggests that the paternal $C d k n 1 c$ allele indeed contributed to the $C d k n 1 c$ transcripts detected with BL6-specific primers in these embryos. We then examined expression of the paternal Cdkn1c allele in NPCs and neurons isolated from the neocortex of E16 embryos derived from a cross between BL6 male and JF1 female mice. These cells were isolated as $\mathrm{CD} 133^{+} \mathrm{CD} 24^{-}$and $\mathrm{CD} 133^{-} \mathrm{CD} 24^{+}$fractions, respectively, by fluorescence-activated cell sorting (FACS), and both were found to express the paternal Cdkn1c allele (Fig. 1b). Together, these results thus indicated that the paternal allele of the $C d k n 1 c$ gene is transcribed, albeit at a low level, in the developing neocortex.

We investigated whether deletion of the paternal $C d k n 1 c$ allele might affect expression of the maternal allele. However, the amounts of maternal or total $C d k n 1 c$ mRNA in the neocortex did not differ significantly among E16 embryos derived from a cross between BL6 male Cdkn1 $c^{\mathrm{f} / \mathrm{fl}}$ and JF1 female Nestin-Cre ${ }^{+/-}$mice (Fig. 1d,e). We also did not detect any significant differences in the total protein level of $\mathrm{p} 57^{\mathrm{kip} 2}$ after the paternal Cdkn1c deletion (Supplementary Fig. 2d).

We next examined the directionality of transcription of $C d k n 1 c$ by performing Northern blot analysis of control brains. As shown in the Supplementary Fig. 2a,b, we detected transcripts only generated from the sense (coding) direction, but not those generated from the antisense direction. We next performed direction (strand)sensitive RNA-sequencing analysis to further examine the directionality of $C d k n 1 c$ transcripts. This analysis again showed that almost all of the reads (1766 out of 1778) that mapped to the Cdkn1c locus were in the sense (coding) direction (Supplementary Fig. 3). At the exon 4 locus at which we conducted allele specific qPCR in Fig. 1, no read was mapped to the antisense strand ( 0 out of 650$)$. This high ratio of transcripts from the sense direction at the exon 4 suggests that transcripts derived from this locus of the paternal allele are also synthesized from the sense (coding) strand similar to the maternal one, although this does not exclude the possibility of antisense expression at the paternal $C d k n 1 c$ allele. We also compared the pattern of $C d k n 1 c$ transcripts between control and paternal $C d k n 1 c$ cKO brain. The pattern of reads that mapped to the $C d k n 1 c$ locus was largely unchanged by paternal $C d k n 1 c$ cKO (Supplementary Fig. 3), again suggestive of the absence (or low abundance) of paternal-specific nonconventional $C d k n 1 c$ transcripts.

We then examined the possibility that the paternal $C d k n 1 c$ deletion may affect other imprinted genes located close to $C d k n 1 c$ in the same gene cluster. We performed RT-qPCR analysis for such genes (Cd81, Phlda2, Osbp15 and Nap1l4) and found that the expression of these genes was not largely affected by the paternal Cdkn1c deletion (Supplementary Fig. $4 \mathrm{a}-\mathrm{d}$ ). We also performed bisulfite sequencing of KvDMR1 and ICG5, located near Cdkn1c, in the control and paternal Cdkn1c cKO neocortices at E16. The levels of DNA methylation at these loci were slightly increased but not overtly affected by paternal $C d k n 1 c \mathrm{cKO}$ (Supplementary Fig. 4e,f). We thus concluded that the levels of imprinting in the same gene cluster were not significantly affected by the paternal Cdkn1c cKO.

CNS-specific paternal Cdkn1c KO reduces the brain size and the number of upper layer neurons at postnatal stage. We next investigated whether the paternal $C d k n 1 c$ allele plays a role in mouse brain development. We deleted the paternal allele in a CNS-specific manner by crossing BL6 Cdkn1 $c^{\mathrm{f} / \mathrm{fl}}$ male with BL6 Nestin-Cre $e^{+-}$female mice and found that such deletion resulted in a substantial reduction in brain size apparent at postnatal day (P) 60 (Fig. 2a). This finding was unexpected given the low level of expression of the paternal allele compared with the maternal allele. Immunohistofluorescence analysis of coronal sections revealed that this size reduction was apparent throughout the entire forebrain-including the neocortex, basal ganglia, thalamus, and septal nuclei-of the paternal cKO mice at P24 (Fig. 2b). The total section size of the paternal Cdkn1c cKO brain at the level of 0.38 to $1.18 \mathrm{~mm}$ relative to the bregma was only $72.37 \pm 6.07 \%$ (mean \pm s.e.m., $n=3$ mice) of that for the control brain at P24. With regard to the neocortex, the mediolateral surface length at this level relative to the bregma was reduced to $81.44 \pm 1.53 \%$ (mean \pm s.e.m., $n=3$ mice) for the paternal $C d k n 1 c$ cKO brain compared with the control brain at P24. The thickness of the primary somatosensory area (S1) of the paternal $C d k n 1 c$ cKO brain was also reduced to $71.33 \pm 10.00 \%$ (mean \pm s.e.m., $n=3$ mice) of that for the control brain at P24.

The forebrain phenotype of the paternal $C d k n 1 c \mathrm{cKO}$ mice appeared largely similar to that of CNS-specific maternal Cdkn1c cKO mice previously generated ${ }^{30}$, although the former was generally less pronounced and showed some distinct features. One such feature of the paternal cKO brain was the apparent absence of hydrocephalus, one of the most remarkable characteristics of the maternal $C d k n 1 c \mathrm{cKO}$ brain $^{30}$. The ventricles of the paternal Cdkn1c cKO brain were not as expanded as those of the control brain at P0 or P24 (Figs. 2b and 3a). The 
a

P60

Control Cdkn1c pKo

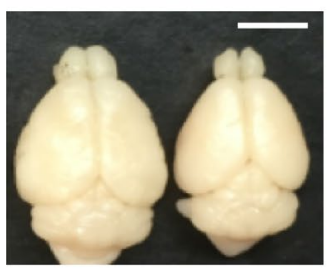

d

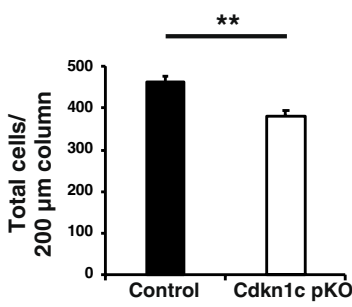

b

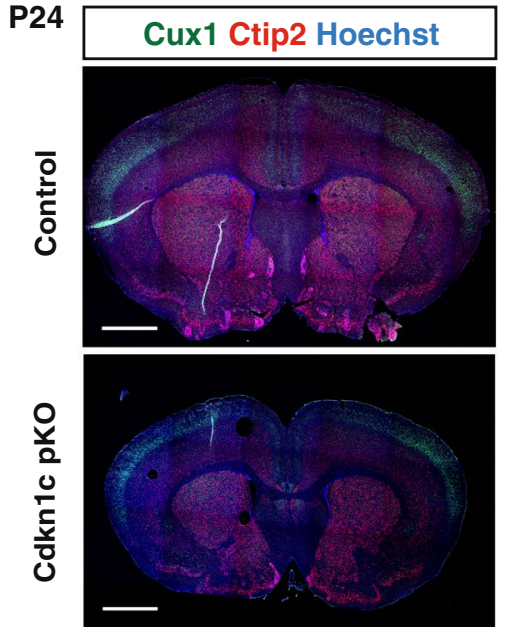

C

\section{P24}

Control Cdkn1c pKo
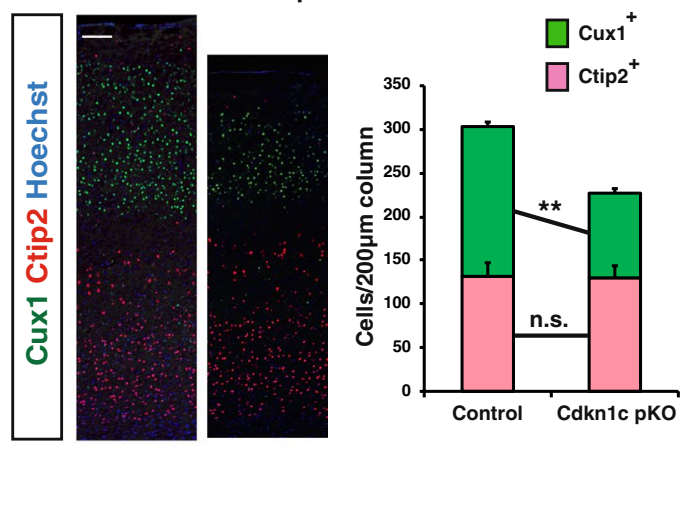

Figure 2. CNS-specific deletion of the Cdkn1c paternal allele reduced the brain size and the number of upper layer neurons at P24. (a-g) Cdkn1c paternal cKO (Nestin-Cre; $\left.C d k n 1 c^{\text {pat flox/++}}\right)$ and control $\left(C d k n 1 c^{\text {pat flox/++}}\right)$ mice were sacrificed at P60 (a) and P24 (b-g). (a) Dorsal view of $C d k n 1 c$ paternal $c K O$ and control brains. (b) Immunofluorescence staining of Cux1 (green) and Ctip2 (red) in coronal sections of Cdkn1c paternal cKO and control mice. Nuclei were stained with Hoechst (blue). (c) Higher magnifications of the neocortical somatosensory area in (b) (left panel). Quantitative analysis of cells positive for Cux1 and Ctip2 per area within $200 \mu \mathrm{m}$ wide bins (right panel) $\left(\mathrm{n}=3\right.$ mice for each genotype). $(\mathbf{d}-\mathbf{g})$ The number of total cells $\left(\mathrm{Hoechst}^{+}\right)(\mathbf{d})$, neurons $\left(\mathrm{NeuN}^{+}\right)(\mathbf{e})$, non-neuronal cells $\left(\mathrm{NeuN}^{-}\right)(\mathbf{f})$ and glial cells $\left(\mathrm{S} 100 \beta^{+}\right)(\mathbf{g})$ per $200 \mu \mathrm{m}$ wide bins in the neocortical area were quantified $(\mathrm{n}=4$ mice for each genotype). Data are mean + s.e.m. Unpaired two-tailed Student's t-test; $* * P<0.01$ n.s., not significant. Scale bars: $500 \mu \mathrm{m}$ in (a); $250 \mu \mathrm{m}$ in (b); $100 \mu \mathrm{m}$ in (c).

previous study of maternal $C d k n 1 c \mathrm{cKO}$ mice concluded that the thinning of the neocortex also apparent in these mice was a result of the hydrocephalus caused by impaired development of the $\mathrm{SCO}^{30}$. Our results revealing a thin neocortex without apparent hydrocephalus in the paternal $C d k n 1 c \mathrm{cKO}$ brain thus indicates that this neocortical phenotype is not due to hydrocephalus, at least not in these animals.

We next examined the neocortex of paternal Cdkn1c cKO mice at P24 in more detail. Consistent with the reduced thickness of the neocortex, the cell number in $\mathrm{S} 1$ was reduced in the paternal $C d k n 1 c \mathrm{cKO}$ mice compared with control mice (Fig. 2d). The number of $\mathrm{NeuN}^{+}$neurons was reduced by paternal Cdkn1c cKO (Fig. 2e) while the number of $\mathrm{NeuN}^{-}$cells and $\mathrm{S} 100 \beta^{+}$astrocytes was not (Fig. 2f,g). Among neurons, the number of $\mathrm{Cux}^{+}$upper layer neurons was significantly reduced whereas that of Ctip2 ${ }^{+}$deep layer neurons was not (Fig. 2c). This selective reduction in the number of upper layer neurons relative to deep layer neurons in the paternal $C d k n 1 c$ cKO neocortex at P24 was confirmed by the detection of a reduced thickness of the upper layers but not of the deep layers in S1 (Supplementary Fig. 5a). We observed similar but severer phenotypes in the maternal Cdkn1c cKO mice at P14 except the reduction of $\mathrm{NeuN}^{-}$cells (Supplementary Fig. 6). Our results together suggested that the paternal $C d k n 1 c$ allele (as well as its maternal allele) might contribute to the genesis of specific neuronal subtypes.

Paternal Cdkn1c cKO reduces the number of neocortical NPCs at late embryonic stages. We next examined when and how paternal $C d k n 1 c \mathrm{cKO}$ results in a reduction in the number of upper layer neurons in the neocortex during development. We first investigated the neocortex of control and paternal $C d k n 1 c \mathrm{cKO}$ mice at $\mathrm{P} 0$ and found that the reduction in the number of $\mathrm{Cux}^{+}$upper layer neurons was already apparent at this stage (Fig. 3a,b). Of note, the number of Ctip $2^{+}$deep layer neurons at this stage was actually increased by paternal $C d k n 1 c$ CKO (Fig. 3b). Consistent with these results, the thickness of the upper layers was reduced but that of the deep layers tended to increase in the paternal Cdkn1c cKO mice at P0 (Supplementary Fig. 5b).

To investigate the mechanism underlying the selective reduction in the number of upper layer neurons in the postnatal neocortex of paternal $C d k n 1 c \mathrm{cKO}$ mice, we evaluated the production of deep layer and upper layer 
a PO

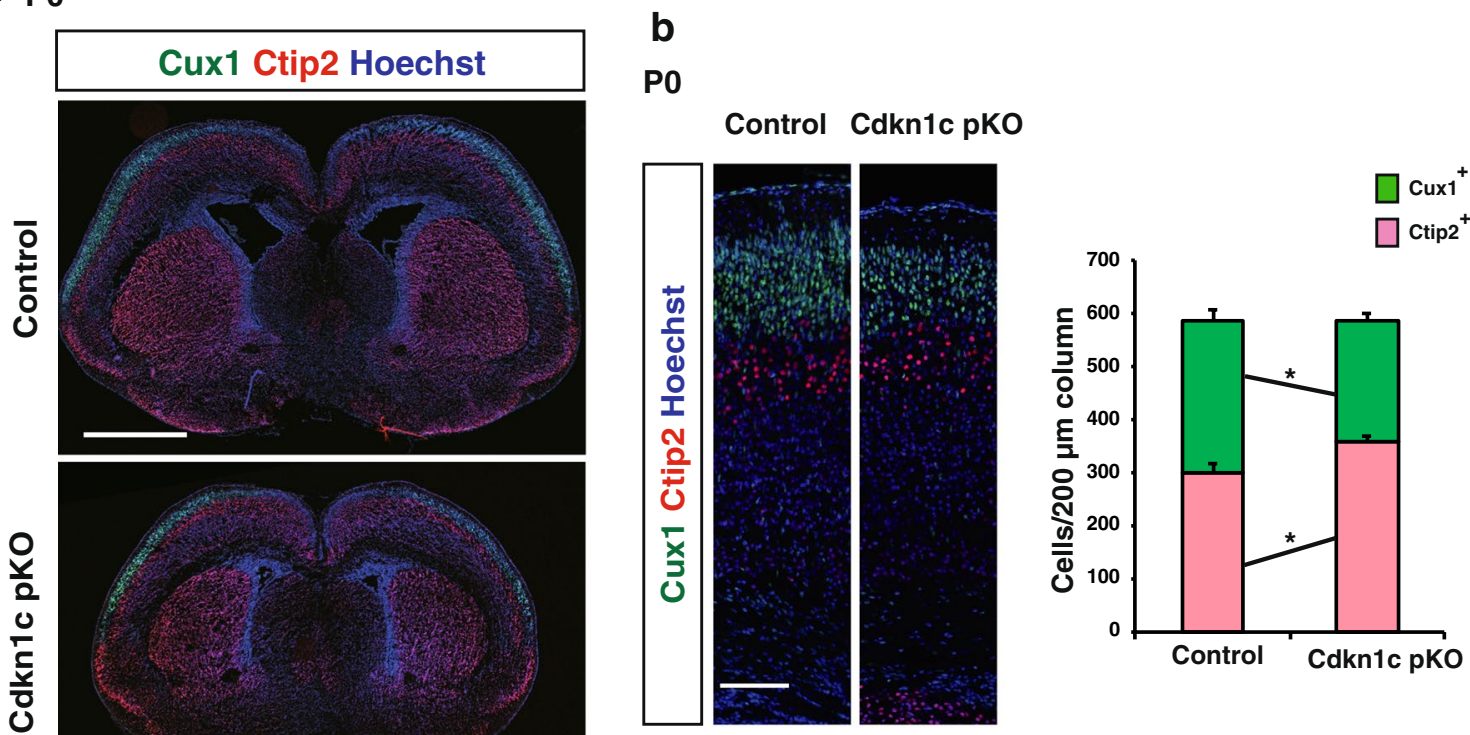

C

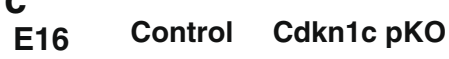

d
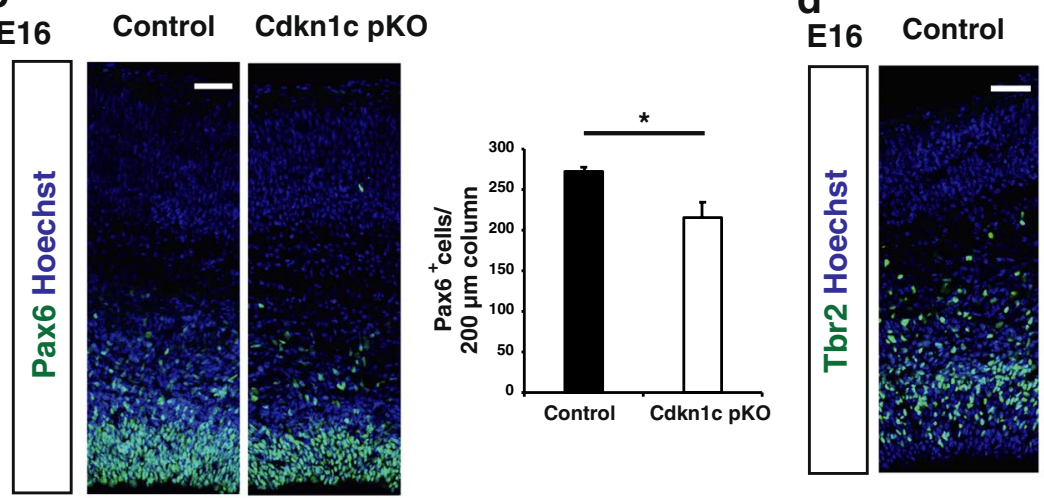

Control

Cdkn1c pKO
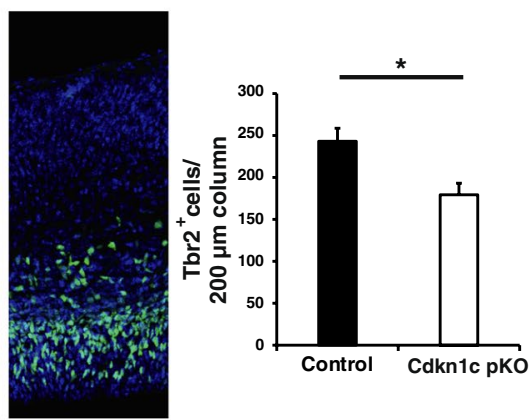

e
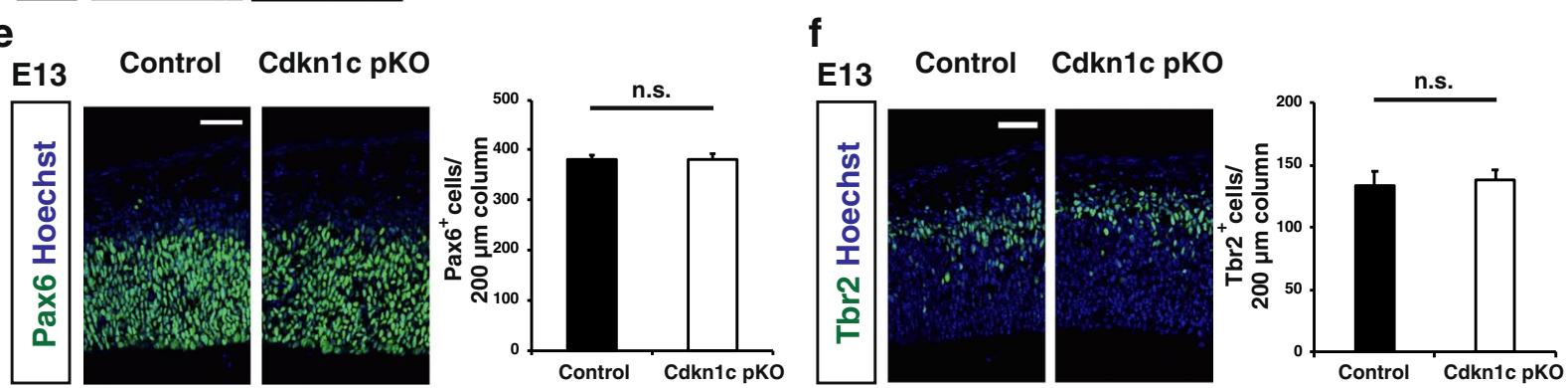

Figure 3. CNS-specific deletion of the $C d k n 1 c$ paternal allele reduced the number of upper layer neurons at $\mathrm{P} 0$ and progenitors at E16. (a) Immunofluorescence staining of Cux1 (green) and Ctip2 (red) in coronal sections of Cdkn1c paternal cKO and control mice at P0. Nuclei were stained with Hoechst (blue). (b) Higher magnifications of neocortical somatosensory area in (a) (left panel). Quantitative analysis of cells positive for Cux1 and Ctip2 per area within $200 \mu \mathrm{m}$ wide bins (right panel) ( $\mathrm{n}=4$ pups for each genotype). (c-f) Immunofluorescence staining of Pax6 (c,e) and Tbr2 (d,f) at E16 (c,d) and E13 (e,f), respectively. The cells positive for Pax6 and Tbr2 per area within $200 \mu \mathrm{m}$ wide bins in the neocortical region were quantified $(\mathrm{n}=4$ embryos for each genotype in $\mathbf{c}, \mathbf{d}$ and $\mathrm{n}=3$ embryos for each genotype in e,f). Data are mean + s.e.m. Unpaired two-tailed Student's t-test; $* P<0.05$. n.s., not significant. Scale bars: $500 \mu \mathrm{m}$ in (a); $100 \mu \mathrm{m}$ in (b); $50 \mu \mathrm{m}$ in (c-f).

neurons at embryonic stages. We first examined the numbers of $\mathrm{Pax}^{+} \mathrm{NPCs}$ and $\mathrm{Tbr} 2^{+}$intermediate neuronal progenitors (INPs) in S1 at E13 and found no significant difference between control and paternal Cdkn1c cKO mice (Fig. 3e,f). However, paternal $C d k n 1 c \mathrm{cKO}$ resulted in a significant reduction in the numbers of both these cell types at E16 (Fig. 3c,d). Consistent with these findings, the number of proliferating cells positive for Ki67 was also reduced in the neocortex of paternal Cdkn1c cKO mice at E16 but not at E13 (Fig. 4a,b). Given that most deep 
a

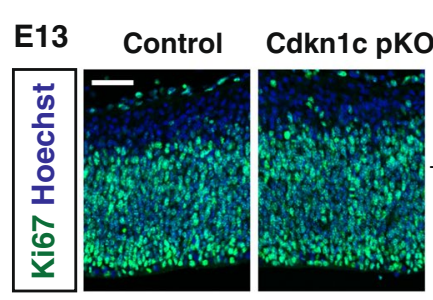

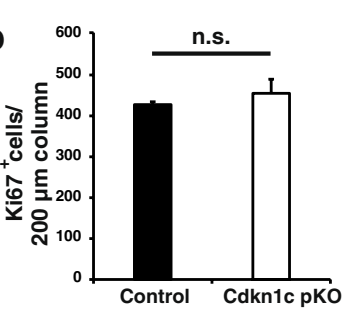

Control Cdkn1c pKo

C
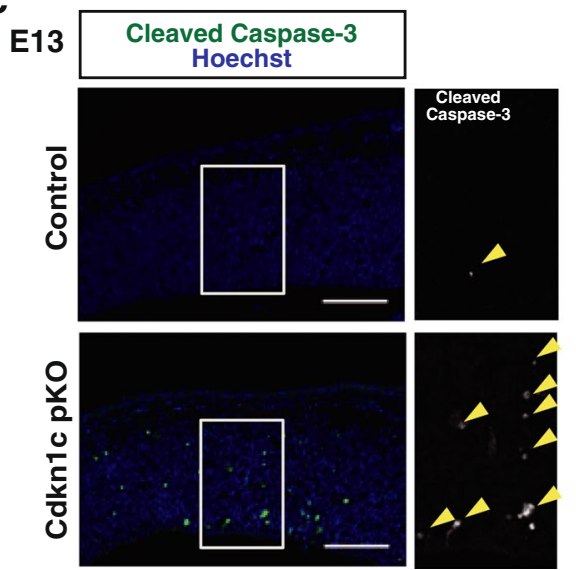

b

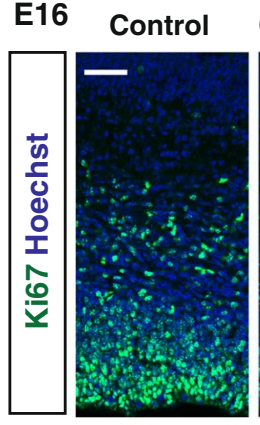

d

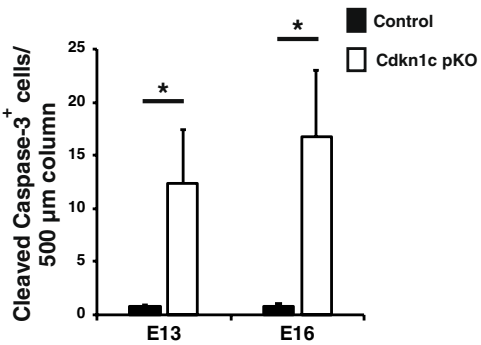

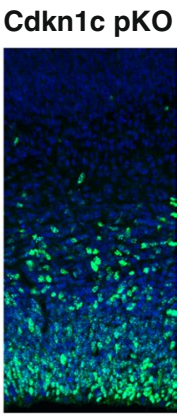

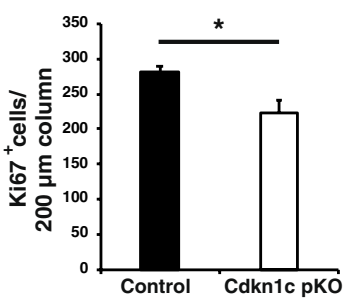

e

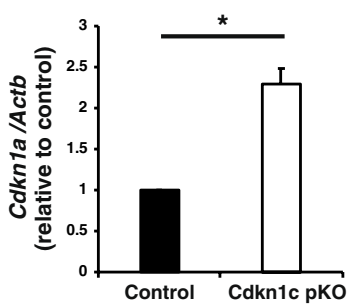

Figure 4. CNS-specific deletion of the $C d k n 1 c$ paternal allele reduced the number of proliferating progenitors at E16 and increased the number of apoptotic cells at E13 and E16. (a,b) Immunofluorescence staining of Ki67 at E13 (a) and E16 (b) (left panel). Quantitative analysis of cells positive for Ki67 per area within $200 \mu \mathrm{m}$ wide bins (right panel) $(n=3-4$ embryos for each genotype). Data are mean + s.e.m. Unpaired two-tailed Student's t-test. (c) Immunostaining of cleaved caspase-3 at E13. The boxed regions in the left panels are shown at a higher magnification in the right panel. Yellow arrowheads indicate cleaved caspase- 3 positive cells. (d) The number of cleaved caspase- 3 positive cells per area within $500 \mu \mathrm{m}$ wide bins were quantified at E13 and E16 $(\mathrm{n}=4-6$ embryos for each genotype). Data are mean + s.e.m. Unpaired two-tailed Student's t-test. (e) qPCR of $C d k n 1 a$ mRNA expression in the neocortex isolated from control and paternal Cdkn1c cKO mice at P0. $C d k n 1 a$ mRNA expression was normalized to $\beta$-actin $(\mathrm{n}=3$ independent experiments). Data are mean + s.e.m.; expressed relative to the corresponding value for control mice. Paired two-tailed Student's t-test. $* P<0.05$. n.s., not significant. Scale bars: $50 \mu \mathrm{m}$ in $(\mathbf{a}, \mathbf{b}) ; 100 \mu \mathrm{m}$ in $(\mathbf{c})$.

layer neurons and upper layer neurons in $\mathrm{S} 1$ are born before and after E13.5, respectively, the differences in the numbers of NPCs and INPs apparent at E16 but not at E13 may explain, at least in part, the selective reduction in the number of upper layer neurons induced by paternal $C d k n 1 c \mathrm{cKO}$. We also found that the number of apoptotic cells positive for the cleaved form of caspase- 3 was increased among NPCs in the neocortex of paternal Cdkn1c cKO mice at both E13 and E16 (Fig. 4c,d). Related to apoptosis induction, we also detected increased expression

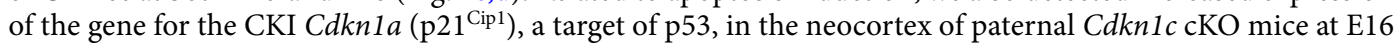
(Fig. 4e). The increase in the extent of cell death might thus account for the reduction in the number of NPCs and subsequently that in INPs in the paternal $C d k n 1 c \mathrm{cKO}$ neocortex. We then performed transcriptomic analysis of the control and paternal Cdkn1c cKO neocortices by RNA sequencing and gene ontology (GO) analysis on differentially expressed genes. Consistent with the increased cell death, we observed enrichment of GO terms such as neuronal death, DNA damage response and signal transduction by 53 class mediator in the upregulated genes by paternal Cdkn1c deletion (Supplementary Fig. 7a). Also, according to the RNA sequencing results, the apoptotic genes such as Ccng1, Trp73 and Sesn 2 were significantly upregulated in paternal Cdkn1c cKO neocortex compared to control. On the other hand, the GO terms enriched in the downregulated genes by paternal Cdkn1c deletion include those related to the mitochondrion pathway such as oxidative phosphorylation (Supplementary Fig. 7b). Together, these results indicate that the paternal $C d k n 1 c$ allele promotes cell survival and plays an important role in the generation of appropriate numbers of NPCs, INPs, and cortical neurons during development.

\section{Discussion}

We have here detected a low level of expression of the paternal Cdkn1c allele in the developing mouse neocortex, in contrast to previous findings that the paternal allele is completely imprinted and silenced ${ }^{20,34}$. Immunohistochemical analysis thus previously showed that the amount of $\mathrm{p} 57^{\mathrm{kip} 2}$ (Cdkn1c) protein was reduced to an undetectable level in the brain by deletion of the maternal Cdkn1c allele ${ }^{34}$, and reporter mice that allow monitoring of the expression of the paternal allele on the basis of the activity of firefly luciferase encoded by 
a construct knocked-in at this locus did not show a luminescence signal under normal dietary conditions ${ }^{34}$. However, in contrast to the allele-specific qPCR analysis adopted in our study, expression of the paternal Cdkn1c allele might have been too low compared with that of the maternal allele to have been detected by these previous methods.

Although expression of the paternal Cdkn1c allele in the brain was $<2 \%$ of that of the maternal allele at E16, we found that paternal $C d k n 1 c \mathrm{cKO}$ resulted in prominent changes to the brain including thinning of the neocortex. Such thinning previously observed in maternal $C d k n 1 c \mathrm{cKO}$ mice was suggested to be a result of defects in the

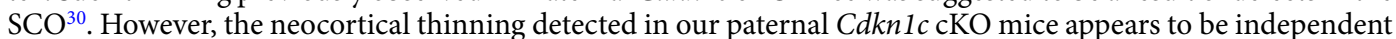
of any effect on the SCO, given the absence of hydrocephalus. Instead, our results suggest that increased cell death and a consequent reduction in the number of neocortical NPCs at the late stage of development give rise to a reduced level of neurogenesis and thinning of the neocortex in paternal Cdkn1c cKO mice. Our results have thus revealed an essential role for the paternal $C d k n 1 c$ allele in control of the survival of neocortical NPCs and the genesis of neurons-in particular, upper layer neurons produced at the late stage of development.

How the paternal $C d k n 1 c$ allele fulfills this role despite its low expression level remains unknown. It is possible that the paternal allele is expressed at similar or even higher levels relative to the maternal allele in a subpopulation of NPCs in which it plays a protective role. Alternatively, the paternal and maternal alleles might confer expression of different mRNA or protein isoforms, although we did not detect any difference in total $C d k n 1 c$ mRNA and protein levels in the absence or presence of the paternal allele. The paternal allele thus does not appear to affect the expression level of the maternal allele. It is also possible that transcripts other than $C d k n 1 c$ mRNA are produced from the paternal $C d k n 1 c$ allele or from cis- or trans-regulatory elements within the paternal $C d k n 1 c$ locus and contribute to the changes to the brain apparent in paternal $C d k n 1 c \mathrm{cKO}$ mice.

Prenatal protein restriction and intrauterine growth retardation have been associated with various psychiatric conditions including schizophrenia, attention-deficit and hyperactivity disorder, autism spectrum disorder, and major affective depression ${ }^{37}$. Of interest in this regard, restriction of maternal dietary protein during pregnancy induces demethylation and aberrant induction of the Cdkn1c locus in the prefrontal cortex and mesolimbic dopaminergic system of the resulting offspring ${ }^{38}$. Indeed, such protein restriction was found to result in permanent derepression of the imprinted paternal Cdkn1c allele through a folate-dependent mechanism of DNA methylation loss ${ }^{34}$. However, whether or how such derepression of the paternal Cdkn1c locus affects brain development remains to be clarified. Given that we found that the paternal $C d k n 1 c$ allele plays an essential role in generation of appropriate numbers of NPCs and neurons, derepression of this allele induced by a low-protein diet may have an impact on NPC proliferation and neurogenesis during neocortical development. We also found that paternal $C d k n 1 c$ cKO resulted in a reduction in the staining intensity for tyrosine hydroxylase $(\mathrm{TH})$ in the striatum (data not shown). Given that $\mathrm{p} 57^{\mathrm{kip} 2}(\mathrm{Cdkn} 1 \mathrm{c})$ has been implicated in the production of $\mathrm{TH}^{+}$dopaminergic neurons ${ }^{27}$ and that twofold overexpression of $C d k n 1 c$ conferred by a transgene both increased TH expression in the brain and altered behaviors that are dependent on the mesolimbic dopaminergic system ${ }^{39,40}$, derepression of the paternal $C d k n 1 c$ locus induced by a low-protein diet might thus lead to behavioral changes through modulation of neurogenesis in both the neocortex and mesolimbic dopaminergic system. The possible role of derepression of the paternal $C d k n 1 c$ allele in the link between early-life adversity and aberrant brain development associated with psychiatric disorders thus warrants further investigation.

\section{Experimental Procedures}

Mice. C57BL6/J (BL6) mice were purchased from CLEA Japan. JF1/Ms (JF1) mice were provided by RIKEN BRC (RBRC00639) through the National BioResource Project of the MEXT/AMED, Japan, and purchased from National Institute of Genetics, Japan. Nestin-Cre mice and Cdkn1c floxed mice were kindly provided by Ryoichiro Kageyama and Keiichi Nakayama, respectively. To generate JF1 Nestin-Cre mice, Nestin-Cre mice were backcrossed to JF1 mice, and confirmed the SNPs at Cdkn1c genomic locus with sanger sequencing. All mice were maintained and studied according to protocols approved by the Animal Care and Use Committee of The University of Tokyo (approval numbers: P25-8, P25-27, PH27-3 and P30-4).

FACS. Neocortex was prepared by manual dissection, digested enzymatically with a papain-based solution (Sumitomo Bakelite) and then resuspended in 0.3\% BSA/PBS containing primary antibodies (PE-conjugated CD133 (mouse, 1:500, BioLegend, 141204) and APC-conjugated CD24 (mouse, 1:500, BioLegend, 101814)). Cells were then sorted with FACS Aria IIIu (BD). Debris and aggregated cells were gated out by forward and side scatter. Gating was done with isotype controls. NPCs and neurons were isolated as CD $133^{+} \mathrm{CD} 24^{-}$fraction and $\mathrm{CD} 133^{-} \mathrm{CD} 24^{+}$fraction, respectively.

RNA extraction and RT-qPCR. Allele specific qPCR was performed on cDNA prepared from sorted cells or neocortical tissues of F1 hybrid mice obtained by crossing BL6 and JF1. For other primer sets, qPCR was carried out with the use of cDNA prepared from neocortical tissues of BL6 mice. Total RNA was extracted using RNAiso Plus (Takara) following the instructions of the manufacturer. Reverse transcription (RT) was performed with at the maximum of $0.5 \mu \mathrm{g}$ of total RNA and ReverTra Ace qPCR Master Mix with gDNA remover (TOYOBO). The obtained cDNA was subjected to real-time PCR analysis in a Roche LightCycler 480 II with THUNDERBIRD SYBR qPCR kit (TOYOBO). As for allele specific qPCR, plasmid DNA containing each SNPs was used as a standard and relative copy number was calculated according to the molecular weight. In other experiments, the amount of mRNA quantified was normalized relative to that of $\beta$-actin mRNA. 
The used primers were as follows:

Actb.

Fw AATAGTCATTCCAAGTATCCATGAAA

Rv GCGACCATCCTCCTCTTAG

Cdkn1a.

Fw CACCTCTAAGGCCAGCTA

Rv AGCAATGTCAAGAGTCGG

Cd81.

Fw GGAAGCTGTACCTCATTGGA

Rv CAGCACCATGCTCAGAATC

Phlda2.

Fw TCACCATCGTCACCAACTA

Rv CGGTTCTGGAAGTCGATCA

Osbp15.

Fw ACCGGCTAAAGATGCTACAA

Rv TGGAGCTTAACATGGTCGAA

Nap1l4.

Fw CGCACATAGAAGCTAAGTTCTAC

Rv TCCTCATTCTCACTGTGCC

Cdkn1c.

Common GGGCAGTACAGGAACCATTTC

BL6 TTAGCTTACAGTGTCCCGCA

JF1 TTAGCTTACAGTGTCCCGTA

Allele specific qPCR primers were designed as previously described ${ }^{41}$. BL6/JF1 SNP sites (BL6 chr7: 143458462, NC_000073, GenBank) were identified using NIG Mouse Genome Database (http://molossinus.nig. ac.jp/msmdb/index.jsp). Total $C d k n 1 c$ mRNA was quantified with common and BL6 primers.

Immunohistochemistry. For immunofluorescence staining, brains were postfixed with ice-cold $4 \%$ (wt/ vol) paraformaldehyde (PFA) at $4{ }^{\circ} \mathrm{C}$ for $2 \mathrm{~h}$, equilibrated with $30 \%(\mathrm{w} / \mathrm{v})$ sucrose in PBS, and frozen in OCT (Tissue TEK). Coronal sections (15-16 $\mu \mathrm{m}$ thickness) were exposed to Tris-buffered saline (TBS) containing $0.1 \%$ Triton X-100 and $2 \%$ Donkey serum (blocking buffer) and incubated overnight at $4{ }^{\circ} \mathrm{C}$ with primary antibodies in blocking buffer and then for $1-2 \mathrm{~h}$ at room temperature with Alexa Fluor-conjugated secondary antibodies in blocking buffer. For staining with the antibody to Cux1, Ctip2, Pax6, Tbr2 and Ki67, we performed antigen retrieval by autoclave treatment of sections with target retrieval solutions (Dako) for $10 \mathrm{~min}$ at $105^{\circ} \mathrm{C}$. Fluorescence images were obtained with a laser confocal microscope (Leica TCS-SP5). Antibodies used for immunostaining included Cux1 (Rabbit, 1:200, Santa Cruz, sc-13024), Ctip2 (Rat, 1:1000, Abcam, ab18465), NeuN (Mouse, 1:200, Millipore, MAB377), S100ß (Rabbit, 1:500, Abcam, AB52642) Pax6 (Rabbit, 1:1000, Millipore, AB2237), Tbr2 (Chicken, 1:1000, Millipore, AB15894), Ki67 (Rat, 1:500, Dako, M7249) cleaved Caspase3 (Rabbit, 1:1000, Cell Signaling, 9664). Alexa-Fluor-labeled secondary antibodies (1:1000) and Hoechst were obtained from Life Technologies.

Western blotting. Total protein was extracted from the neocortex of P0 pups with the use of sonication. Lysates were prepared in $1 \times$ Laemmli's buffer and loaded in each well of a $10 \%$ acrylamide gel. Following transfer, the membranes were blocked with $2 \%$ skim milk in Tris-buffered saline containing $0.05 \%$ Tween 20 and incubated overnight $\left(4^{\circ} \mathrm{C}\right)$ with the following primary antibodies: $57^{\mathrm{kip} 2}(\mathrm{Cdkn} 1 \mathrm{c})$ (Rabbit, 1:500, Sigma-Aldrich, P0357); $\beta$-actin (Mouse, 1:5000, Sigma-Aldrich, A2228). To examine a loading control, blots were stripped and reanalyzed for $\beta$-actin. The images were acquired by ImageQuant LAS4000 (GE Healthcare) and quantified using ImageQuant TL (GE Healthcare).

Northern blotting. One $\mu$ g of total RNAs in a denaturing buffer were run on a $1 \%$ agarose gel, transferred and chemically crosslinked to Hybond-N (GE Healthcare). 28 S RNA was detected with methylene blue. After washing, membranes were hybridized with probes in DIG Easy Hyb Granules (Sigma Aldrich) at $65^{\circ} \mathrm{C}$. The region encoding $C d k n 1 c$ in the plasmid ${ }^{42}$ was transcribed with T3 promoter (antisense probe) and T7 promoter (sense probe), labeled with DIG RNA Labelling Mix (Roche) and used as probes. The images were acquired by ImageQuant LAS4000 (GE Healthcare) and quantified using ImageQuant TL (GE Healthcare).

Bisulfite analysis. Bisulfite treatment of the genomic DNA isolated from neocortex at E16 was performed using the EpiTect bisulfite kit (Qiagen). Each of KvDMR and ICG5 was amplified by PCR, and the products were subcloned and sequenced. The primer to amplify KvDMR1 (ICG8b) was previously described ${ }^{17}$. For ICG5, PCR amplification of the promoter region of $C d k n 1 c$ was carried out using primers below.

Fw GGAGTTGAAGGATTAGTTTTTTT

Rv ATATAAACATTTCCCCTTATCCC

RNA sequencing analysis. cDNA libraries were prepared using total RNA from neocortical tissues at E16 according to the Tru-seq library construction protocol. The library products were then sequenced by Illumina NovaSeq6000 (100 bp paired-end, stranded). Raw sequences were aligned to the mouse mm 10 genome by 
HISAT ${ }^{43}$ using a transcriptome index built from RefSeq mm10 and visualized with the IGV browser. We tested for differential gene expression using edgeR and defined differentially expressed genes as having an adjusted p-value of less than 0.05 . Functional annotation of the dysregulated genes (GO analysis) was performed with Metascape $^{44}$. RNA-seq data is deposited at DDBJ (accession no. DRA009463).

Statistical analysis. Data are presented as means + s.e.m. as indicated, and were analysed by Student's two-tailed paired $t$ test and Student's two-tailed unpaired $t$ test, as indicated. A $P$ value of $<0.05$ was considered statistically significant and the significance is marked by $* P<0.05$ and $* * P<0.01$. The number of animals in each experiment is stated in the respective figure legends.

Received: 3 June 2019; Accepted: 16 January 2020;

Published online: 05 February 2020

\section{References}

1. John, R. M. \& Surani, M. A. Genomic imprinting, mammalian evolution, and the mystery of egg-laying mammals. Cell 101, 585-588, https://doi.org/10.1016/S0092-8674(00)80870-3 (2000).

2. Reik, W. \& Walter, J. Evolution of imprinting mechanisms: The battle of the sexes begins in the zygote. Nat. Genet. 27, 255-256, https://doi.org/10.1038/85804 (2001).

3. Ferguson-Smith, A. C. Genomic imprinting: The emergence of an epigenetic paradigm. Nat. Rev. Genet. 12, 565-575, https://doi. org/10.1038/nrg3032 (2011).

4. Uribe-Lewis, S., Woodfine, K., Stojic, L. \& Murrell, A. Molecular mechanisms of genomic imprinting and clinical implications for cancer. Expert Rev. Mol. Med. 13, 1-22, https://doi.org/10.1017/S1462399410001717 (2011).

5. Radford, E. J., Ferrón, S. R. \& Ferguson-Smith, A. C. Genomic imprinting as an adaptative model of developmental plasticity. FEBS Lett. 585, 2059-2066, https://doi.org/10.1016/j.febslet.2011.05.063 (2011).

6. Peters, J. The role of genomic imprinting in biology and disease: An expanding view. Nat. Rev. Genet. 15, 517-530, https://doi. org/10.1038/nrg3766 (2014).

7. Demars, J. \& Gicquel, C. Epigenetic and genetic disturbance of the imprinted 11p15 region in Beckwith-Wiedemann and SilverRussell syndromes. Clin. Genet. 81, 350-361, https://doi.org/10.1111/j.1399-0004.2011.01822.x (2012).

8. Buiting, K. Prader-Willi syndrome and Angelman syndrome. Am. J. Med. Genet. Part C Semin. Med. Genet. 154, 365-376, https:// doi.org/10.1002/ajmg.c.30273 (2010).

9. Crowley, J. J. et al. Analyses of allele-specific gene expression in highly divergent mouse crosses identifies pervasive allelic imbalance. Nat. Genet. 47, 353-360, https://doi.org/10.1038/ng.3222 (2015).

10. Ferrón, S. R. et al. Postnatal loss of Dlk1 imprinting in stem cells and niche astrocytes regulates neurogenesis. Nature 475, 381-385, https://doi.org/10.1038/nature10229 (2011).

11. Ferrón, S. R. et al. Differential genomic imprinting regulates paracrine and autocrine roles of IGF2 in mouse adult neurogenesis. Nat. Commun. 6, 8265, https://doi.org/10.1038/ncomms9265 (2015).

12. Hatada, I. \& Mukai, T. Genomic imprinting of p57KIP2, a cyclin-dependent kinase inhibitor, in mouse. Nat. Genet. 11, 204-206, https://doi.org/10.1038/ng1095-204(1995).

13. Matsuoka, S. et al. Imprinting encoding cyclin-dependent kinase inhibitor, p57KIP2,on chromosome 11p15. Genetics 93, 3026-3030 (1996).

14. Bhogal, B., Arnaudo, A., Dymkowski, A., Best, A. \& Davis, T. L. Methylation at mouse Cdkn1c is acquired during postimplantation development and functions to maintain imprinted expression. Genomics 84, 961-970, https://doi.org/10.1016/j.ygeno.2004.08.004 (2004).

15. Fitzpatrick, G. V., Soloway, P. D. \& Higgins, M. J. Regional loss of imprinting and growth deficiency in mice with a targeted deletion of KvDMR1. Nat. Genet. 32, 426-431, https://doi.org/10.1038/ng988 (2002).

16. Mancini-DiNardo, D., Steele, S. J. S., Levorse, J. M., Ingram, R. S. \& Tilghman, S. M. Elongation of the Kenq1ot1 transcript is required for genomic imprinting of neighboring genes. Genes Dev. 20, 1268-1282, https://doi.org/10.1101/gad.1416906 (2006).

17. Fan, T. et al. Lsh controls silencing of the imprinted Cdknlc gene. Development 132, 635-644, https://doi.org/10.1242/dev.01612 (2005).

18. Lee, M. H., Reynisdttir, I. \& Massague, J. Cloning of p57KIP2, a cyclin-dependent kinase inhibitor with unique domain structure and tissue distribution. Genes Dev. 9, 639-649, https://doi.org/10.1101/gad.9.6.639 (1995).

19. Matsuoka, S. et al. p57KIP2, a structurally distinct member of the p21CIP1 Cdk inhibitor family, is a candidate tumor suppressor gene. Genes Dev. 650-662, https://doi.org/10.1101/gad.9.6.650 (1995).

20. Zhang, P. et al. Altered cell differentiation and proliferation in mice lacking p57KIP2 indicates a role in Beckwith-Wiedemann syndrome. Nature 387, 151-158, https://doi.org/10.1038/387151a0 (1997).

21. Westbury, J., Watkins, M., Ferguson-Smith, A. C. \& Smith, J. Dynamic temporal and spatial regulation of the cdk inhibitor p57k2 during embryo morphogenesis. Mech. Dev. 109, 83-89, https://doi.org/10.1016/S0925-4773(01)00512-3 (2001).

22. Hoovers, J. M. et al. Multiple genetic loci within 11 p15 defined by Beckwith-Wiedemann syndrome rearrangement breakpoints and subchromosomal transferable fragments. Proc. Natl. Acad. Sci. USA 92, 12456-12460 (1995).

23. Hatada, I. et al. An imprinted gene $\mathrm{p} 57^{\mathrm{KIP} 2}$ is mutated in Beckwith-Wiedemann syndrome. Genes Dev. 11, 973-983, https://doi. org/10.1038/ng1096-171 (1996).

24. Yan, Y., Frisén, J., Lee, M. H., Massagué, J. \& Barbacid, M. Ablation of the CDK inhibitor p57 Kip2 results in increased apoptosis and delayed differentiation during mouse development. Genes Dev. 11, 973-983, https://doi.org/10.1101/gad.11.8.973 (1997).

25. Takahashi, K. \& Nakayama, K. Mice lacking a CDK inhibitor, p57Kip2, exhibit skeletal abnormalities and growth retardation. J. Biochem. 127, 73-83 (2000).

26. Itoh, Y., Masuyama, N., Nakayama, K., Nakayama, K. I. \& Gotoh, Y. The cyclin-dependent kinase inhibitors p57 and p27 regulate neuronal migration in the developing mouse neocortex. J. Biol. Chem. 282, 390-396, https://doi.org/10.1074/jbc.M609944200 (2007).

27. Joseph, B. et al. p57(Kip2) cooperates with Nurr1 in developing dopamine cells. Proc. Natl. Acad. Sci. USA 100, 15619-15624, https://doi.org/10.1073/pnas.2635658100 (2003).

28. Joseph, B. et al. p57 $57^{\text {Kip2 }}$ is a repressor of Mash1 activity and neuronal differentiation in neural stem cells. Cell Death Differ. 16, 1256-1265, https://doi.org/10.1038/cdd.2009.72 (2009).

29. Tury, A., Mairet-Coello, G. \& Dicicco-Bloom, E. The cyclin-dependent kinase inhibitor p57Kip2 regulates cell cycle exit, differentiation, and migration of embryonic cerebral cortical precursors. Cereb. Cortex 21, 1840-1856, https://doi.org/10.1093/ cercor/bhq254 (2011)

30. Matsumoto, A. et al. Deregulation of the p57-E2F1-p53 Axis Results in Nonobstructive Hydrocephalus and Cerebellar Malformation in Mice. Mol. Cell. Biol. 31, 4176-4192, https://doi.org/10.1128/mcb.05370-11 (2011). 
31. Furutachi, S., Matsumoto, A., Nakayama, K. I. \& Gotoh, Y. P57 controls adult neural stem cell quiescence and modulates the pace of lifelong neurogenesis. EMBO J. 32, 970-981, https://doi.org/10.1038/emboj.2013.50 (2013).

32. Mairet-Coello, G. et al. p57KIP2 regulates radial glia and intermediate precursor cell cycle dynamics and lower layer neurogenesis in developing cerebral cortex. Development 139, 475-487, https://doi.org/10.1242/dev.067314 (2012).

33. Furutachi, S. et al. Slowly dividing neural progenitors are an embryonic origin of adult neural stem cells. Nat. Neurosci. 18, 657-665, https://doi.org/10.1038/nn.3989 (2015).

34. Van de Pette, M. et al. Visualizing Changes in Cdkn1c Expression Links Early-Life Adversity to Imprint Mis-regulation in Adults. Cell Rep. 18, 1090-1099, https://doi.org/10.1016/j.celrep.2017.01.010 (2017).

35. Bouschet, T. et al. In Vitro Corticogenesis from Embryonic Stem Cells Recapitulates the In Vivo Epigenetic Control of Imprinted Gene Expression. Cereb. Cortex 27, 2418-2433, https://doi.org/10.1093/cercor/bhw102. (2017).

36. Isaka, F. et al. Ectopic expression of the bHLH gene Math1 disturbs neural development. Eur. J. Neurosci. 11, 2582-2588, https://doi. org/10.1046/j.1460-9568.1999.00699.x (2003).

37. O'Neil, A. et al. Preventing mental health problems in offspring by targeting dietary intake of pregnant women. BMC Med. 12, 1-7, https://doi.org/10.1186/s12916-014-0208-0 (2014).

38. Vucetic, Z. et al. Early life protein restriction alters dopamine circuitry. Neuroscience 168, 359-370, https://doi.org/10.1016/j. neuroscience.2010.04.010 (2010).

39. McNamara, G. I., Davis, B. A., Dwyer, D. M., John, R. M. \& Isles, A. R. Behavioural abnormalities in a novel mouse model for Silver Russell Syndrome. Hum. Mol. Genet. 25, 5407-5417, https://doi.org/10.1093/hmg/ddw357 (2016).

40. Mcnamara, G. I. et al. Dopaminergic and behavioural changes in a loss-of-imprinting model of Cdkn1c. Genes, Brain Behav. 149-157, https://doi.org/10.1111/gbb.12422 (2017).

41. Gaudet, M., Fara, A.-G., Beritognolo, I., \& Sabatti, M. Allele-Specific PCR. In: Komar A. Single Nucleotide Polymorphisms. Methods in Molecular Biology ${ }^{\mathrm{TM}}$ (Methods and Protocols). 578. Humana Press, Totowa, NJ (2009)

42. Suzuki-Hirano, A. et al. Dynamic spatiotemporal gene expression in embryonic mouse thalamus. J. Comp. Neurol. 519, 528-543, https://doi.org/10.1002/cne.22531 (2011).

43. Kim, D., Paggi, J. M., Park, C., Bennett, C. \& Salzberg, S. L. Graph-based genome alignment and genotyping with HISAT2 and HISAT-genotype. Nat. Biotechnol. 37, 907-915 (2019).

44. Zhou, Y. et al. Metascape provides a biologist-oriented resource for the analysis of systems-level datasets. Nat. Commun. 10 (2019).

\section{Acknowledgements}

We thank Toshihiko Shiroishi for providing us the genomic information of the JF1/Ms mouse strain; Keiichi Nakayama and Tsunaki Higa for providing Cdkn1c floxed mice; Ryoichiro Kageyama for providing Nestin-Cre mice; Yuki Katou and other members of Shirahige laboratory for help with sequencing analysis; Seth Blackshaw for providing the probe used for Northern blotting; and all members of the Gotoh laboratory for discussion and support. This study was supported by KAKENHI grants from the Ministry of Education, Culture, Sports, Science, and Technology of Japan and the Japan Society for the Promotion of Science (JP17J08651 for Y.I.; JP18K06477 for D.K.; JP16H06481, JP16H06279, JP16H06479, JP15H05773 for Y.G.) as well as by AMED-CREST of the Japan Agency for Medical Research and Development (JP19gm0610013 and JP19gm1310004), by the Uehara Memorial Foundation and by the International Research Center for Neurointelligence (WPI-IRCN) at The University of Tokyo Institutes for Advanced Study.

\section{Author contributions}

Y.I. performed the experiments, analyzed the data, and wrote the manuscript. T.W. designed the allele specific qPCR of $C d k n 1 c$. H.M. conducted initial analysis of $C d k n 1 c$ paternal $c K O$ mice. D.K., S.F. and Y.G. conceived of and coordinated the project as well as wrote the manuscript. All authors approved the final version of the manuscript.

\section{Competing interests}

The authors declare no competing interests.

\section{Additional information}

Supplementary information is available for this paper at https://doi.org/10.1038/s41598-020-58629-9.

Correspondence and requests for materials should be addressed to D.K. or Y.G.

Reprints and permissions information is available at www.nature.com/reprints.

Publisher's note Springer Nature remains neutral with regard to jurisdictional claims in published maps and institutional affiliations.

(c) (i) Open Access This article is licensed under a Creative Commons Attribution 4.0 International

License, which permits use, sharing, adaptation, distribution and reproduction in any medium or format, as long as you give appropriate credit to the original author(s) and the source, provide a link to the Creative Commons license, and indicate if changes were made. The images or other third party material in this article are included in the article's Creative Commons license, unless indicated otherwise in a credit line to the material. If material is not included in the article's Creative Commons license and your intended use is not permitted by statutory regulation or exceeds the permitted use, you will need to obtain permission directly from the copyright holder. To view a copy of this license, visit http://creativecommons.org/licenses/by/4.0/.

(C) The Author(s) 2020 